\title{
The Effect of Neurofeedback on Brain Waves in Children With Autism Spectrum Disorders
}

\author{
Sahel Hemmati ${ }^{1}$, Roshanak Vameghi ${ }^{1 *}$, Firoozeh Sajedi ${ }^{1}$, Masoud Gharib ${ }^{2}$, Masoume Pourmohammadreza-Tajrishi ${ }^{1}$, Robab Teymori $^{1}$ \\ 1. Pediatric Neurorehabilitation Research Center, University of Social Welfare and Rehabilitation Sciences, Tehran, Iran. \\ 2. Department of Rehabilitation, Faculty of Paramedical Sciences, Mazandran University of Medical Sciences, Sari, Iran.
}

dtation: Hemmati S, Vameghi R, Sajedi F, Gharib M, Pormohammadreza-Tajrishi M, Teymori1 R. The Effect of Neurofeedback on Brain Waves in Children With Autism Spectrum Disorders. Iranian Rehabilitation Journal. 2016; 14(3):133-138. https://doi.org/10.18869/nrip.irj.14.3.133

: https://doi.org/10.18869/nrip.irj.14.3.133

Article info:

Received: 24 Jan. 2016

Accepted: 08 Jul. 2016

\section{Keywords:}

Autism Spectrum Disorders (ASD), Neurofeedback, EEG waves

\section{A B S T RACT}

Objectives: Autism Spectrum Disorder (ASD) is a group of neurodevelopment syndromes with impairments in social communications and restrictive or repetitive behaviors. Neurofeedback is a new method that may regulate neuro and metabolic function in brain. This study is semiexperimental, with pre and post test.

Methods: In 26 children with ASD, 40 sessions of Neurofeedback were done. Brain waves were recorded before and after intervention by EEG/ERP 19 channels.

Results: The average of Theta (low frequency) power decreased, but there was no difference in the Gamma of Gamma waves (High frequency).

Discussion: Our findings showed that the implementation of Neurofeedback sessions improved Theta, which leads to better social communication.

\section{Introduction}

A

utism Spectrum Disorder (ASD), previously known as the Pervasive Developmental Disorder (PDD), is a phenotypically heterogeneous group of neurodevelopment syndromes [1]. Autistic Disorder (AD) was characterized by impairment in two domains: social communities and restricted and repetitive behaviors that present in the early developmental period. However, when subtle, these symptoms may not be identified until several years later [2]. ASD is typically evident during the second year of life and in severe cases; a lack of developmentally appropriate interests in social interactions may be noted even in the first year [3]. Some studies suggest that a decline in social interaction may ensue between the first and second years of life [4]. Although language impairment is not a core diagnostic criteria in ASD, delayed language is often accompanied by diminished social behavior [5].

ASD is associated with several biomarkers, potentially resulting from interactions of gens and environmental factors, which then influence neuronal function, dendrite development, and contribute to altered neuronal information processing [6].

In terms "Theory of mind" that represented Mirror Neuron System (MNS) and Atypical pattern of frontal lobe

* Corresponding Author:

Roshanak Vameghi, MD

Address: Pediatric Neurorehabilitation Research Center, University of Social Welfare and Rehabilitation Sciences, Koodakyar Ave., Daneshjoo Blvd.,

Evin, Tehran, Iran.

Tel: +98 (21) 22180099

E-mail: rvamegi@yahoo.com 
activation [7]. Past researches showed fractal dimension of EEG, as a key characteristic of brain dynamics, and the complexity of brain dynamics has been found to be associated with EEG abnormalities [8]. It occur with greater than expected frequency in ASD [9]. Basic researches have shown abnormally high amount of theta and beta activity at central and parital lobes that are related to attention and autistic behavior [10]. Gamma activity is related to socialization aspects such as problem solving and is associated with abnormality in ASD [11].

Neurofeedback refers to a form of operant conditioning of electrical brain activity, in which desirable brain activity is rewarded and undesirable brain activity is inhibited [12]. The goal of treatment in ASD is to improve social interactions and communications and increase long-term skills in independent living. Neurofeedback has increased social interaction by providing computer games in which the desired behavior is reinforced [13].

The aim of Neurofeedback is to influence brain wave activity to prolong or produce electrical activity during desired behaviors [14]. Neurofeedback that is based on adjusting the degree of connectivity in different areas of the brain has been found to be particularly powerful in reducing symptoms of Autism [15]. In this research, study Neurofeedback effects in the rhythms of brain and behavior.

\section{Methods}

This research was an experimental study with pre and post test. Children with ASD that visited the University rehabilitation center in 1392 were included in the study. The inclusion criteria were 5-18 years of age, visited by psychiatrist for ASD diagnosis, IQ score (60-75) for doing tasks, no seizure or other serious medical illness, and parents should be available to fill the consent for research. None of the included patient used psychiatric drugs. Legal constancy was approved by the ethics committee in this research.

Tools in this research were demographic and personal child questionnaire and Raven test, EEG/ERP with 19 canals in frequency $250 \mathrm{HZ}$. CARS is a questioner scale for detecting autistic behaviors [16]. It has scales in communication (verbal and non verbal), imitations, repetitive behavior, and body posturing. A therapist scales the items in the test from 1.5- 40. The average number is 15-60. ASD disorders are those items scored with 30 or above [17]. In 1385, Rafei translated the test to Persian and Dehkordi was showed 0.92 alpha Cronbach and $\mathrm{T}=0.83$ [18]. Executive function is approved by Stroop - test. Reliability of Persian form was 0.75 , and validity of the test was 0.76 .
In this study, Theta and Gamma activities were studied. The findings were analyzed using specific mathematical methods. Sessions were done in the mornings after the subjects had their breakfast. The participants also did not suffer from any other illness like common cold, etc. At first, the brain signal in resting position was monitored ( 3 minutes with open and closed eyes). Then the brain signal was monitored when performing standard tasks.

The time given for each task was about 1 hour. According to the Autism protocol, 40 Neurofeedback sessions of 30-45 minutes each were conducted twice a week. Theta and Gamma activities were visualized in separate bar graphics using the computer screen. Power values of each frequency band were calculated. Pre-processing procedures were done for removing and minimizing noises and artifacts: low-pass filter, high-pass filter, and notch filter $(50 \mathrm{HZ})$.

EEG/ERP, signals assessment by mathematics method (linear and non - linear) like: IAFs, ITFs, Functional Community, Complexity Dimensions, DFA Coefficient, Coherence patterns, Fractal Dimensions. ANOVA model and orbital radius classifier were done for detecting differences. Moreover, the efficacy of Neurofeedback sessions was estimated for each individual subject by calculating linear regression and Spearman's regression coefficient fitting the progression of power values over sessions. The effects of Neurofeedback in the EEG were studied by rhythm analysis and adjusted with the behavioral questionnaire.

\section{Results}

In this study, 30 children (5-18 Y) with ASD were participated and utility 26 children were cooperated. As shown in Figure 1, the theta wave activity (low frequency) after Neurofeedback decreased, which could be a sign of improvement. CARS questionnaire was used before and after 40 sessions. It was found that there was an increase in the activity after Neurofeedback. Figure 2 shows that the gamma waves' activity (high frequency) after Neurofeedback did not change.

Figure 3 shows that the average of Theta waves was higher than the control in most channels before Neurofeedback treatment but after Neurofeedback, these waves decreased. This finding is showed response treatment to Neurofeedback intervention. In Figure 4, the Gamma activities were mostly similar to that of the normal children, and after Neurofeedback, there seems to be no difference between the groups. Utility after intervention in all channels Theta activity was raised that shows behavioral improvement (at first $\mathrm{CARS}=45$, finally=35). 


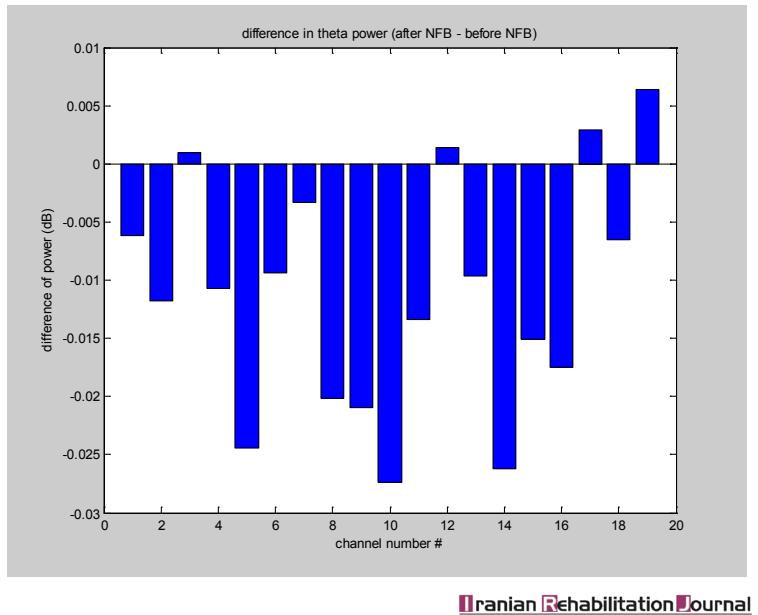

Figure 1. Average of (power after NFB - power before NFB) overall the subjects in each channel.

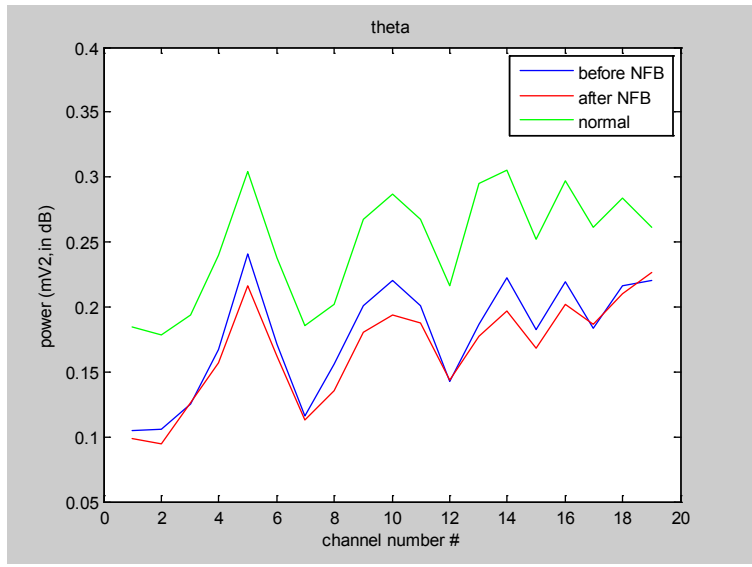

Ilranian Rehabilitation Journa

Figure 3. average of Theta waves before and after Neurofeedback treatment with control.

\section{Discussion}

The present study evaluated the effects of Neurofeedback training for the treatment of children with ASD. Neurofeedback is believed to elicit growth and changes at the cellular levels of the brain activity, which in turn supports brain functioning and behavioral cognitive performance [12]. Reduction of Theta was hypothesized to improve children's executive function. Cognitive improvement due better executive function received. Moreover, past researches were showed in Cerebral palsy, relation between the deficient brain dynamics and severity of disabilities [19] and trying on Delta and Alfa brain waves are showing improvement in them [20]. Consistent with our prediction, the children with ASD made larger improvement in this area after Neurofeedback training. Mirjam Kojzer in 2009 reported that Neurofeedback protocol training helped make improve-

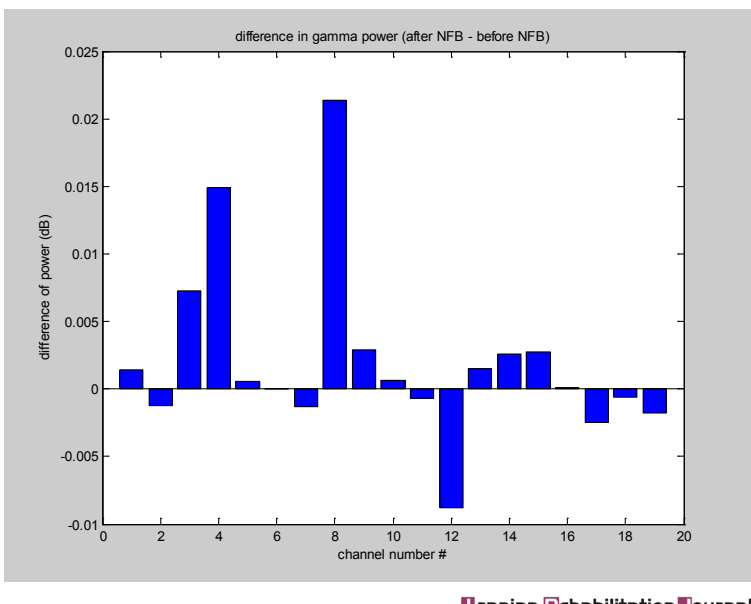

Figure 2. Gamma activity (High frequency). This figure are average of (power after NFB - power before NFB) overall the subjects in each channel.

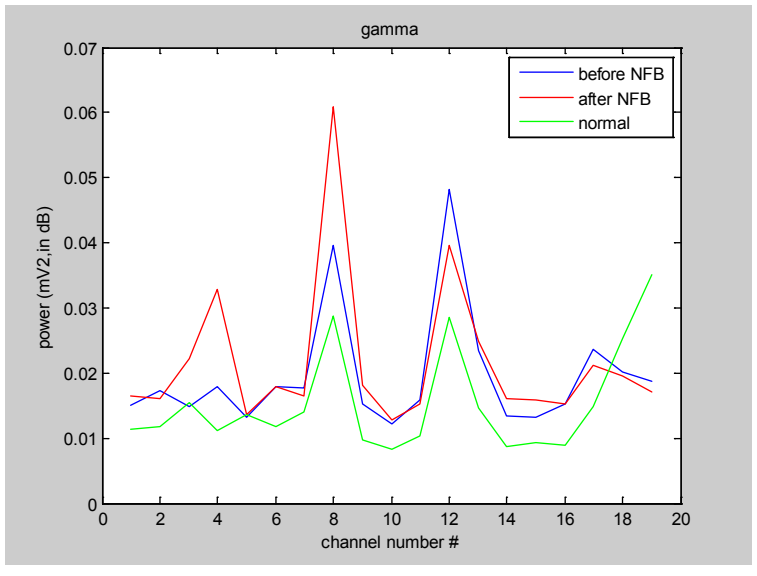

Ilranian Rehabilitation Journal

Figure 4. average of Gamma waves before and after Neurofeedback treatment with control.

ment in ASD patients and reduce theta power and delta overtime [21]. Koropotov and colleagues in 2007 found no notable changes in QEEG power spectra in ADHD, although Neurofeedback training was found to affect the amplitude components. In 2007, another study evaluated executive functioning of children with ASD after Neurofeedback training using a questionnaire completed by parents and teachers. Their findings are in agreement with the present study results that there is a significant improvement in reducing theta and executive function [14]. High functioning autistic children showed elevated theta power [9]. Some findings provided a possible mechanism through the relation between theta power, executive function, and social communication [22].

Gamma related the perceptual and cognitive task, which is deficit in ASD. Our study showed that there was no difference in Gamma power after Neurofeed- 
back training. Macado showed increased gamma in autism [23]. A similar number of studies, however, have observed a decrease in Gamma. Another study observed lower gamma in autism when compared with normal children and after Neurofeedback remained [24].

\section{Conclusion}

CARS show improvement after Neurofeedback sessions. This finding shows that although Neurofeedback does not decrease the Gamma, it improves the ASD activity.

Further clinical work and research should focus on possible synergistic effects of Neurofeedback and other interventions. We view Neurofeedback as an intervention that may prove to be efficacious in the treatment of symptoms of autism. In conclusion, the application of a typical ADHD Neurofeedback protocol to a group of ASD children was found to be highly affective, especially in executive function. It is necessary to further investigate the efficacy of this method in the treatment of ASD.

\section{Acknowledgements}

The current research hasn't received any financial support.

\section{Conflict of Interest}

The authors declared no conflict of interests.

\section{References}

[1] Hodo DW. Kaplan and Sadock's synopsis of psychiatry: behavioral sciences clinical psychiatry. Journal of the American Medical Association. 1996; 275(11):883-84. doi: 10.1001/ jama.1996.03530350065041

[2] Maenner MJ, Rice CE, Arneson CL, Cunniff C, Schieve LA, Carpenter LA, et al. Potential impact of DSM- 5 criteria on autism spectrum disorder prevalence estimates. JAMA Psychiatry. 2014; 71(3):292-300. doi: 10.1001/jamapsychiatry.2013.3893

[3] Coben R, Linden M, Myers TE. Neurofeedback for autistic spectrum disorder: a review of the literature. Applied Psychophysiology and Biofeedback. 2010; 35(1):83-105. doi: 10.1007/ s10484-009-9117-y

[4] Ben-Itzchak E, Zachor DA. The effects of intellectual functioning and autism severity on outcome of early behavioral intervention for children with autism. Research in Developmental Disabilities. 2007; 28(3):287-303. doi: 10.1016/j. ridd.2006.03.002
[5] American Psychiatric Association. Diagnostic and statistical manual of mental disorders; Washington, D. C.: American Psychiatric Association; 1980.

[6] Kuś R, Kamiński M, Blinowska KJ. Determination of EEG activity propagation: Pair-wise versus multichannel estimate. IEEE Transactions on Biomedical Engineering. 2004; 51(9):1501-510. doi: 10.1109/tbme.2004.827929

[7] Baron-Cohen S, Leslie AM, Frith U. Does the autistic child have a "theory of mind"? Cognition. 1985; 21(1):37-46. doi: 10.1016/0010-0277(85)90022-8

[8] Hemmati S, Ahmadlou M, Gharib M, Vameghi R, Sajedi F. Down syndrome's brain dynamics: analysis of fractality in resting state. Cognitive Neurodynamics. 2013; 7(4):333-40. doi: 10.1007/s11571-013-9248-y

[9] Murias M, Webb SJ, Greenson J, Dawson G. Resting state cortical connectivity reflected in EEG coherence in individuals with autism. Biological Psychiatry. 2007; 62(3):270-73. doi: 10.1016/j.biopsych.2006.11.012

[10] Kouijzer ME, de Moor JM, Gerrits BJ, Congedo M, van Schie HT. Neurofeedback improves executive functioning in children with autism spectrum disorders. Research in Autism Spectrum Disorders. 2009; 3(1):145-62. doi: 10.1016/j. rasd.2008.05.001

[11] Higgins JP, Thompson SG, Deeks JJ, Altman DG. Measuring inconsistency in meta-analyses. British Medical Journal. 2003; 327(7414):557-60. doi: 10.1136/bmj.327.7414.557

[12] Demos JN. Getting started with neurofeedback. New York: W. W. Norton \& Company; 2005.

[13] Evans JR. Handbook of neurofeedback: dynamics and clinical applications. Boca Ranton: CRC Press; 2007.

[14] Oberman LM, Ramachandran VS, Pineda JA. Modulation of mu suppression in children with autism spectrum disorders in response to familiar or unfamiliar stimuli: the mirror neuron hypothesis. Neuropsychologia. 2008; 46(5):1558-565. doi: 10.1016/j.neuropsychologia.2008.01.010

[15] Coben R, Padolsky I. Assessment-guided neurofeedback for autistic spectrum disorder. Journal of Neurotherapy. 2007; 11(1):5-23. doi: 10.1300/j184v11n01_02

[16] Castelloe P, Dawson G. Subclassification of children with autism and pervasive developmental disorder: A questionnaire based on Wing's subgrouping scheme. Journal of Autism \& Developmental Disorders. 1993; 23(2):229-41. doi: $10.1007 /$ bf01046217

[17] Robins DL, Fein D, Barton ML, Green JA. The modified checklist for autism in Toddlers: An initial study investigating the early detection of autism and pervasive developmental disorders. Journal of Autism \& Developmental Disorders. 2001; 31(2):131-44. PMID: 11450812

[18] Dehkordi Reasi S, Rias RM. Using mobile game application to teach children with Autism Spectrum Disorder (ASD) multiple cues responding: A pilot study. Paper Presented at: The $3^{\text {rd }}$ International Conference on IEEE; 2014 Sep 2-5; Shah Alam, Malaysia.

[19] Sajedi F, Ahmadlou M, Vameghi R, Gharib M, Hemmati S Linear and nonlinear analysis of brain dynamics in children with cerebral palsy. Research in Developmental Disabilities. 2013; 34(5):1388-396. doi: 10.1016/j.ridd.2013.01.016 
[20] Ahmadlou M, Gharib M, Hemmati S, Vameghi R, Sajedi F. Disrupted small-world brain network in children with Down Syndrome. Clinical Neurophysiology. 2013; 124(9):1755-764. doi: 10.1016/j.clinph.2013.03.004

[21] Jensen O, Tesche CD. Frontal theta activity in humans increases with memory load in a working memory task. European Journal of Neuroscience. 2002; 15(8):1395-399. doi: 10.1046/j.1460-9568.2002.01975.x

[22] Machado J. Early childhood experiences in language arts: Early literacy. Boston, Massachusetts: Cengage Learning; 2015.

[23] Amin OSM, Abdullah AA, Xaznadar A, Shaikhani M Striatocapsular infarction; A single institutional experience. Acta Informatica Medica. 2012; 20(2):106-12. doi: 10.5455/ aim.2012.20.106-112

[24] Mirab-Zadeh A. [Review: Attention and Memory in Autism (Persian)]. Rehabilitation Journal. 2001; 2(3-4):51-56. 
